\title{
Efetividade de dois métodos explicativos para pacientes pediátricos sobre a proporção de dentifrícios
}

\author{
Effectiveness of two explanatory methods for pediatric patients on the proportion of \\ toothpaste
}

Efectividad de dos métodos explicativos para pacientes pediátricos sobre la proporción de pasta dental

Samylla Bezerra Rodrigues ${ }^{1 *}$, Kauana da Silva Andrade ${ }^{1}$, Fábio Victor Dias Silva ${ }^{1}$, Vitória da Silva Lima $^{1}$, Bianca Maria de Melo Costa ${ }^{1}$, Consuelo Fernanda Macedo de Souza ${ }^{1}$, Carlus Alberto Oliveira dos Santos ${ }^{2}$, Cristiane Araújo Maia Silva ${ }^{3}$, Bianca Oliveira Tôrres ${ }^{1}$, Fernanda de Araújo Trigueiro Campos ${ }^{1}$.

\begin{abstract}
RESUMO
Objetivo: Avaliar a eficácia de dois métodos explicativos acerca da quantidade de dentifrício fluoretado. Métodos: Trata-se de um estudo de caráter transversal, intervencional, descritivo e quantitativo, com uma amostra de 80 pacientes, de 5 a 10 anos de idade, atendidos em uma clínica escola de Odontologia em João Pessoa - PB. Os pacientes foram randomizados em quatro grupos: grupo controle, grupo com acesso à explicação oral, enquanto outro grupo teve acesso apenas a explicação escrita e um grupo com a explicação associada. Resultados: Entre os participantes da pesquisa, $59,5 \%$ eram do sexo feminino e $40,5 \%$ do sexo masculino, com idade média de 7,59 anos. No teste estatístico de Kruskal Wallis, verificou-se que não há diferença estatística em relação ao método educativo utilizado entre os grupos, enquanto o teste de Wilcocxon demonstrou diferença entre os demais grupos quando comparados com o grupo controle, onde o método associado se mostrou mais eficaz. No teste de Friedman, constatou-se, mais uma vez, que o método associado se mostrou mais eficiente. Conclusão: De acordo com os resultados encontrados, observou-se que não há diferença estatística em relação ao método educativo utilizado entre os grupos de crianças, entretanto, outros testes demonstraram que o método associado apresentou melhores resultados.
\end{abstract}

Palavras-chave: Odontopediatria, Dentifrícios, Flúor.

\section{ABSTRACT}

Objective: To evaluate the effectiveness of two explanatory methods about the amount of fluoride toothpaste. Methods: This is a cross-sectional, interventional, descriptive and quantitative study, with a sample of 80 patients, from 5 to 10 years old, seen at a dental school clinic in João Pessoa - PB. Patients were randomized into four groups: control group, group with access to oral explanation, while another group had access only to written explanation and a group with associated explanation. Results: Among the survey participants, 59.5\% were female and $40.5 \%$ male, with an average age of 7.59 years. In the Kruskal Wallis statistical test, it was found that there is no statistical difference in relation to the educational method used between the groups, while the Wilcocxon test demonstrated difference between the other groups when compared with the control group, where the associated method was more effective. In the Friedman test, it was found, once again, that the associated method proved to be more efficient. Conclusion: According to the results found, it was observed that there is no statistical difference in relation to the educational method used between the groups of children, however, other tests showed that the associated method showed better results.

Keywords: Pediatric dentistry, Dentifrices, Fluorine.

${ }^{1}$ Centro Universitário de João Pessoa (UNIPÊ), João Pessoa - PB.

2 Universidade Estadual da Paraíba (UEPB), Campina Grande - PB.

${ }^{3}$ Centro Universitário UNIESP, João Pessoa - PB.

*E-mail: samyllabr@gmail.com

SUBMETIDO EM: 6/2020 


\section{RESUMEN}

Objetivo: Evaluar la efectividad de dos métodos explicativos con respecto a la cantidad de pasta dental con fluoruro. Métodos: Se trata de un estudio transversal, intervencionista, descriptivo y cuantitativo, con una muestra de 80 pacientes, de 5 a 10 años, atendidos en una clínica de odontología en João Pessoa - PB. Los pacientes fueron aleatorizados en cuatro grupos: grupo de control, grupo con acceso a explicación oral, mientras que otro grupo tuvo acceso solo a explicación escrita y un grupo con explicación asociada. Resultados: Entre los participantes de la encuesta, 59.5\% eran mujeres y $40.5 \%$ hombres, con una edad promedio de 7.59 años. En la prueba estadística de Kruskal Wallis, se encontró que no hay diferencia estadística en relación con el método educativo utilizado entre los grupos, mientras que la prueba de Wilcocxon demostró la diferencia entre los otros grupos en comparación con el grupo de control, donde el método asociado era más eficaz. En la prueba de Friedman, se encontró, una vez más, que el método asociado demostró ser más eficiente. Conclusión: De acuerdo con los resultados encontrados, se observó que no existe una diferencia estadística en relación con el método educativo utilizado entre los grupos de niños, sin embargo, otras pruebas mostraron que el método asociado mostró mejores resultados.

Palabras clave: Odontología pediátrica, Dentífricos, Flúor.

\section{INTRODUÇÃO}

A cárie dentária e a doença periodontal são preocupantes problemas de saúde pública, pois são as enfermidades bucais mais frequentemente encontradas, que podem levar a perda do elemento dental se o tratamento precoce e adequado não for realizado (FILHO PA, et al., 2014; SILVA-JUNIOR MF, et al., 2017).

Os dados encontrados na Pesquisa Nacional de Saúde Bucal (SB) Brasil de 2010, mostraram que aos cinco anos de idade, uma criança brasileira possui dentes cariados, perdidos e obturados, sendo uma média de 2,43. Comparando esse valor com o obtido no SB Brasil de 2003, que foi de 2,8, observa-se uma redução de apenas $13,9 \%$ em um período de sete anos.

Comparando os resultados do SB Brasil 2003 e 2010 para o índice de dentes cariados, perdidos e obturados (CPO-D) aos 12 anos, observa-se que o valor passou de 2,78 em 2003 para 2,07 em 2010, correspondendo a uma redução de $26,2 \%$ em sete anos.

Dos dentes avaliados em relação a experiência de cárie, observou-se um predomínio da doença cárie, responsável por mais de $80 \%$ do índice total, demonstrando um valor percentual significativo para as crianças (LOPES LM, et al., 2014).

Apesar da Odontologia ter apresentado grandes avanços, principalmente no que diz respeito ao surgimento de novos materiais restauradores e técnicas de regeneração do periodonto, os métodos preventivos por meio mecânico continuam sendo importantes aliados no combate à cárie e as doenças periodontais (DUQUE AD, et al., 2020).

A escovação dentária é considerada um dos principais métodos preventivos das patologias bucais e o que oferece um melhor impacto, uma vez que, desorganiza o biofilme dentário, que é o responsável, tanto pela desmineralização dos tecidos dentários como pela destruição dos tecidos de sustentação.

Além de ser um método de fácil acesso a população e bastante efetivo, quando realizada adequadamente. A escovação está associada aos dentifrícios, substâncias químicas terapêuticas, que melhoraram a saúde bucal da população (MAGALHÃES AC, et al., 2011).

A efetividade dos dentifrícios fluoretados na redução da cárie dentária é bastante difundida, pois ao realizar a escovação com esse material ocorre a desorganização do biofilme e assegura a permanência do flúor na cavidade bucal. Porém, a concentração desses dentifrícios deve ser levada em consideração, uma vez que, quando a concentração for inferior a 1000 ppm não ocorre a proteção contra a cárie (SILVA MF, et al., 2019). 
A prevenção da cárie e da doença periodontal ocorre pelo controle mecânico do biofilme dental por meio da utilização da escova convencional, do uso de dentifrícios fluoretados e do fio dental, associados às visitas periódicas ao cirurgião-dentista.

Embora estudos clínicos mostrem a eficácia desse método na prevenção das doenças bucais, o controle do biofilme não é garantido em todos os casos, sendo esse um recurso de eficácia de curto prazo, pois esses procedimentos, aplicados de forma isolada através de conhecimentos inadequados e sem as orientações realizadas pelo profissional não irão promover um resultado satisfatório (DUQUE AD, et al., 2020).

Sendo assim, a motivação associada ao processo de conscientização deve ser iniciada durante a infância, pois é nesse período que o processo educativo é efetivo por ser a fase de desenvolvimento intelectual da criança.

Dessa forma, as crianças registram um maior aprendizado, eternizando consigo conhecimentos e hábitos que estimularão os cuidados com a saúde bucal e que serão reproduzidas nas fases seguintes da vida (SOUSA JB, et al., 2017).

A principal mudança de hábito que deve ser estimulada e melhorada é o correto controle do biofilme bacteriano por meio das medidas de higiene oral (BALLESTRERI R, et al., 2016). Desse modo, além de se ensinar as técnicas corretas e eficientes de escovação e uso do fio dental, é importante educar e motivar o paciente acerca da importância de tais procedimentos para a manutenção da saúde bucal.

Para o atendimento à população infantil, os métodos utilizados para a educação e motivação dessas crianças, além de eficazes, duráveis e de fáceis utilização, devem ser atraentes e despertar a atenção das crianças para que assim resultados melhores sejam obtidos (SOUSA JB, et al., 2017).

Frente ao exposto, o objetivo desse trabalho foi avaliar a eficácia de dois métodos explicativos acerca da quantidade de dentifrício na clínica escola de Odontologia do Centro Universitário de João Pessoa - PB, onde foram avaliados a efetividade dos métodos de explicação verbal e escrita sobre a quantidade de dentifrício, e comparar, quantitativamente, o dentifrício colocado nas escovas pelas crianças após serem submetidas a esses métodos.

\section{MÉTODOS}

Trata-se de uma pesquisa transversal, intervencional, descritiva e quantitativa realizada na clínica escola do curso Odontologia de uma Instituição de Ensino Superior, localizada em João Pessoa, na Paraíba. A pesquisa foi aprovada com CAAE 02343418.6.0000.516 e parecer consubstanciado de número 3.187.912.

A amostra foi constituída por 80 pacientes pediátricos, de 5 a 10 anos de idade, que estivessem sendo atendidos, no período de 2019.1 e 2019.2, nas clínicas das disciplinas de Odontopediatria I e Odontopediatria II.

Os critérios de elegibilidade utilizados foram pacientes em atendimento inicial na clínica de Odontopediatria com idade entre 5 e 10 anos, cujos pais ou responsáveis tenham assinado o Termo de Consentimento Livre e Esclarecido (TCLE) assentindo a participação na pesquisa.

Enquanto os critérios de exclusão preconizados foram pacientes que se sentiram constrangidos ou desistiram do consentimento fornecido, além de crianças com algum tipo de deficiência seja sensorial, motora ou cognitiva.

Os participantes foram randomizados em quatro grupos, de acordo com a ordem de comparecimento na clínica, sendo o grupo I com instruções orais acerca do uso correto da quantidade de creme dental, o grupo II com instruções por escrito com um panfleto educativo e ilustrativo sobre o uso correto da quantidade de creme dental (Figura 1). Enquanto o grupo III teve a associação dos dois métodos anteriores e grupo IV, o grupo controle, foi formado pelas crianças sem intervenção. 
Figura 1 - Panfleto utilizado para a orientação sobre a quantidade adequada de creme dental.

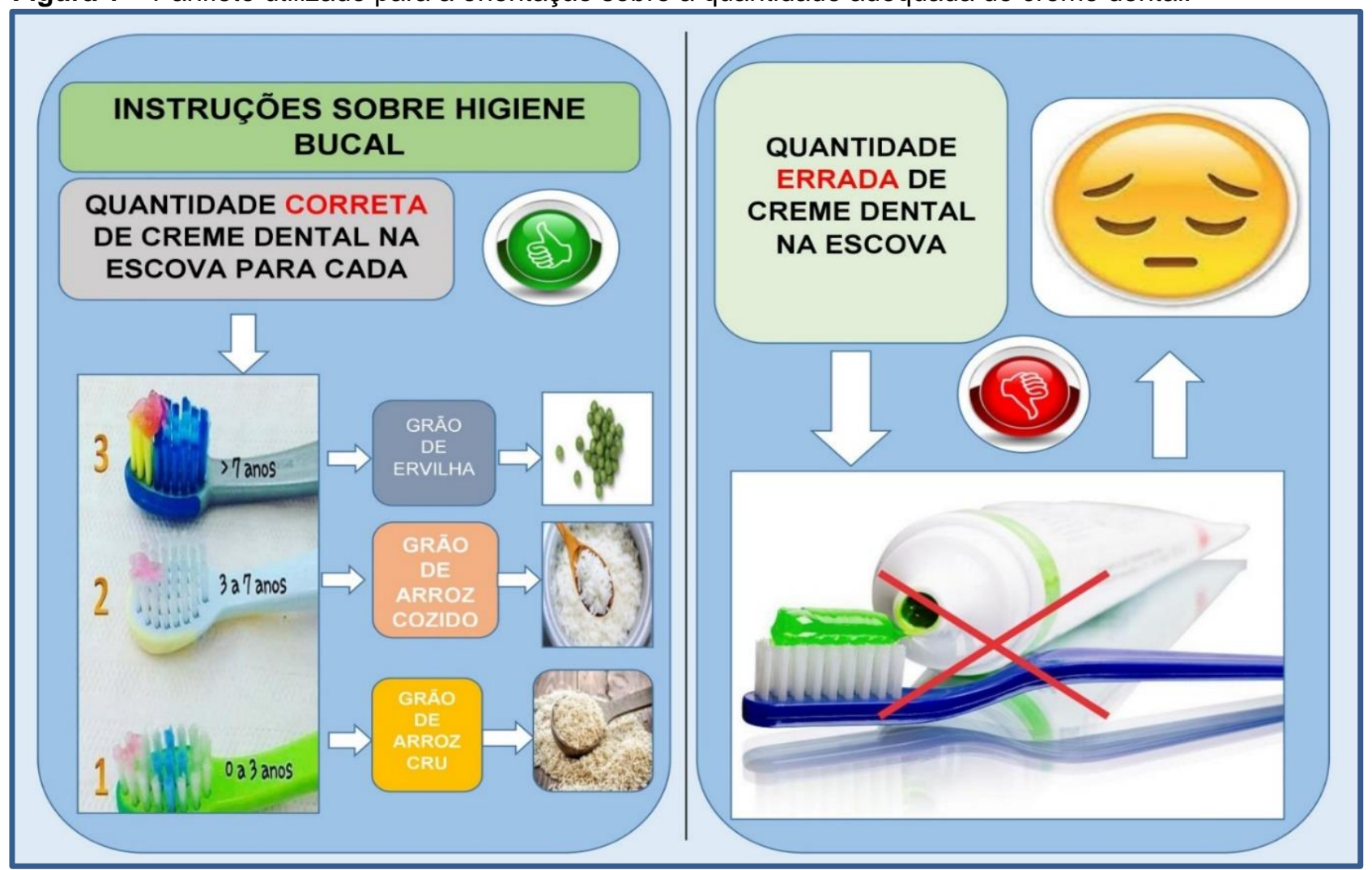

Fonte: Rodrigues SB, et al., 2020.

Inicialmente, as crianças foram orientadas sobre a escovação dentária, sendo colocado a quantidade de creme dental sobre a escova antes da intervenção. Em seguida, as escovas com o creme dental foram pesadas em uma balança de precisão devidamente calibrada e a quantidade registrada foi anotada na ficha de avaliação.

Após a intervenção, de acordo com os métodos descritos anteriormente, as crianças foram instruídas a dispensarem o conteúdo do creme dental sobre a escova e em seguida foi pesada, mais uma vez, em balança de precisão para fins de comparação. Foi utilizada uma ficha de avaliação constando a idade e o sexo do participante, o método de explicação utilizado (oral ou por escrito), bem como uma seção para anotação do peso do creme dental colocado pela criança na escova dental.

Os pesquisadores envolvidos foram previamente calibrados e instruídos a adotarem a mesma linguagem verbal, controle de voz e abordagem, evitando possíveis vieses. Os dados coletados foram armazenados na forma de banco de dados do programa Statistical Package for Social Sciences (SPSS) para Windows, versão 20.0 e analisados por meio de estatística descritiva.

\section{RESULTADOS}

A amostra da pesquisa foi constituída por 80 crianças, atendidas nas clínicas de Odontopediatria I e Odontopediatria II do curso de Odontologia. Em relação ao sexo dos participantes, foi observado que 59,5\% das crianças eram do sexo feminino e $40,5 \%$ do sexo masculino (Tabela 1).

Tabela 1 - Percentual de acordo com o sexo dos participantes da pesquisa. João Pessoa - PB, 2019.

\begin{tabular}{cc}
\hline Sexo & Percentual (\%) \\
\hline Masculino & $40,5 \%$ \\
Feminino & $59,5 \%$ \\
\hline
\end{tabular}

Fonte: Rodrigues SB, et al., 2020. 
Quanto a idade dos pacientes pediátricos, os participantes apresentavam idades em um intervalo de 5 a 10 anos de idade, sendo uma média 7,59 anos (Tabela 2).

Tabela 2 - Idade (em anos) das crianças participantes da pesquisa. João Pessoa - PB, 2019.

\begin{tabular}{ccc}
\hline Idade minima & Idade máxima & Média das idades \\
\hline $\mathbf{5}$ & 10 & 7,59 \\
\hline
\end{tabular}

Fonte: Rodrigues SB, et al., 2020.

No teste estatístico de Kruskal Wallis, os grupos foram comparados entre si. Nesta análise, foi observado que não houve diferença estatística em relação ao método utilizado entre os grupos de crianças que receberam o método oral, o método escrito e o método associado. Dessa forma, quando essas intervenções foram aplicadas, permitiram resultados positivos em relação a quantidade adequada de creme dental a ser utilizada pelas crianças (Tabela 3).

Tabela 3 - Aplicação do teste de Kruskal Wallis para demonstrar a diferença estatística entre os métodos aplicados após a intervenção. João Pessoa - PB, 2019.

\begin{tabular}{lcccc} 
(P-valor sig. > ou $=0,05)$ & Método oral & Método escrito & Método associado & Grupo Controle \\
\cline { 2 - 5 } & 0,202 & 0,156 & 0,309 & 0,537
\end{tabular}

Fonte: Rodrigues SB, et al., 2020.

Os resultados estatísticos do teste de Wilcocxon demonstraram que houve diferença entre os grupos que receberam instruções orais, explicações escritas através de panfletos e associação dos dois métodos, orientações orais e escritas, quando comparados com o grupo controle, constituído por crianças sem instruções. Associado a isso, foi constatado que o método associado foi o mais eficiente (Tabela 4).

Tabela 4 - Dados após aplicação do teste de Wilcocxon. Comparação dos métodos aplicados com o grupo controle. João Pessoa - PB, 2019.

\begin{tabular}{ccccc}
\hline (P-valor sig. > ou $=0,05)$ & Método oral & Método escrito & Método associado & Grupo Controle \\
\cline { 2 - 5 } & 0,202 & 0,156 & 0,309 & 0,537 \\
\hline
\end{tabular}

Fonte: Rodrigues SB, et al., 2020.

O teste de Friedman, onde foram realizadas análises com significância estatística, acerca da eficácia dos métodos explicativos a partir da comparação realizada antes e após a sua aplicação. A partir deste teste, observou-se, estatisticamente, diferenças entre os grupos, sendo o método associado considerado como o mais eficaz (Tabela 5).

Tabela 5 - Dados após aplicação do teste de Friedman. Análise com significância estatística comparando antes e após a intervenção. João Pessoa - PB, 2019.

\begin{tabular}{lllllll}
\hline & \multicolumn{3}{c}{ Método oral } & \multicolumn{2}{c}{ Método escrito } & \multicolumn{2}{c}{ Método associado } \\
\cline { 2 - 7 } & Antes & Depois & Antes & Depois & Antes & Depois \\
\cline { 2 - 7 } & 4,82 & 2,45 & 4,16 & 2,63 & 4,55 & 2,39 \\
\hline
\end{tabular}

Fonte: Rodrigues SB, et al., 2020.

\section{DISCUSSÃO}

Entre os processos que acontecem nas superfícies dentárias, os processos de desmineralização e remineralização (DES/RE) são fundamentais para a manutenção da saúde bucal. Isso só é possível devido ao flúor em atividade, presente na saliva, durante todo o processo da doença cárie, e não apenas pela incorporação de flúor em baixas concentrações no esmalte. O flúor pode ser encontrado nos dentifrícios e nas águas tratadas ofertadas por alguns governos, levando em questão a saúde pública e o potencial efeito cariostático, ambos com abrangências semelhantes. 
Atualmente, recomenda-se o uso de dentifrício fluoretado a partir da erupção do primeiro dente decíduo, apesar de não existir uma padronização da quantidade exata a ser depositada na escova, alguns trabalhos demonstram que em crianças, com menos de 7 anos de idade, pequenas doses (cerca de $0,3 \mathrm{~g}$ ) podem ser administradas (CURY JA, et al., 2001; JAGHER AC, et al., 2016).

A Associação Brasileira de Odontopediatria afirma que para ter uma ação anti-cárie é necessário apenas uma pequena quantidade de dentifrício, desde que contenha a concentração de 1000-1100 ppm. Com isso, previne-se a cárie dentária e assegura-se em relação ao risco de fluorose. O flúor não deve ser abolido da higiene bucal das crianças, devido ao seu benefício anti-cárie, e, sim, utilizado racionalmente. No entanto, os profissionais de Odontologia devem realizar e orientar ações de promoção de saúde orientando os pais e responsáveis para a supervisão da escovação das crianças (LAZZARIN HC, et al., 2018).

De acordo com Silva DAS, et al. (2017), o acompanhamento dos pais ou responsáveis durante a higienização bucal das crianças torna-se essencial, pois a maioria delas ainda não apresenta uma precisa habilidade manual, com isso, elas podem exagerar na quantidade de dentifrício, assim como degluti-lo. Camatta IB e Oliveira CS (2019) realizaram um estudo objetivando avaliar o conhecimento dos familiares sobre a higienização bucal e o uso domiciliar de dentifrício fluoretado de crianças atendidas em uma clínica escola.

Dentre os seus achados, $93,5 \%$ dos pais não sabiam a concentração de fluoreto presente no creme dental utilizado pelas crianças e $60,9 \%$ deles não sabiam o que poderia ocorrer a partir da ingestão do dentifrício fluoretado. Além disso, concluiu-se que muitos responsáveis conhecem a quantidade de creme dental que deve ser utilizada, entretanto, não compreendem, totalmente, os riscos do desenvolvimento da fluorose dentária.

Entre as evidências científicas disponíveis acerca das estratégias para o controle da cárie, é recomendado, atualmente, que um creme dental fluoretado de, no mínimo, 1100 ppm de flúor, seja utilizado, pelo menos, duas vezes ao dia como coadjuvante na limpeza dos dentes de todas as crianças, especialmente direcionada aqueles elementos dentários com alto risco de cárie como, por exemplo, os primeiros molares permanentes em processo de erupção (JAGHER AC, et al., 2016).

O uso de dentifrícios fluoretados para crianças é sempre indicado, uma vez que, a utilização dos cremes dentais sem o flúor resulta em uma população com maior índice de cárie dentária (CASTILHO LS, et al., 2016). No entanto, é importante ressaltar que conforme especificado pelo guia de recomendações para uso de fluoretados no Brasil, recomenda-se que a população, especialmente as crianças menores de nove anos de idade, devem usar os dentifrícios fluoretados em pequenas quantidades devido ao risco de fluorose dentária (OLIVEIRA CC, et al., 2018).

De acordo com a literatura, existe uma "idade crítica" quanto ao risco de fluorose, que está compreendida entre os primeiros meses de vida e os 4 anos de idade, pois o excesso de fluoreto atinge e afeta o dente préerupcionado, e nessas idades, as crianças ainda não possuem um reflexo de deglutição completamente desenvolvido, ou seja, com menos controle nessa questão, há um maior risco de ingestão de dentifrício fluoretado, sendo inversamente proporcional a idade da criança. Dessa forma, quanto mais jovem for a criança, maior é o risco da ingestão de dentifrício (LIMA MNT, et al., 2018).

Lima-Arsati YBO, et al. (2018) buscaram em seu estudo determinar a exposição ao fluoreto em crianças, na idade crítica para a fluorose dentária, residentes no semi-árido do Brasil, tendo como fontes de flúor, a dieta e o dentifrício. Os seus resultados evidenciaram que 19,2\% dos crianças foram expostas a uma dose igual ou superior ao limite de $0,07 \mathrm{mg} \mathrm{F} / \mathrm{kg}$ apenas pelo flúor do creme dental, e 70,5\% do dentifrício colocado sobre a escova foi deglutido durante a escovação. Em contrapartida, Miranda FP e Cericato GO (2016) observaram que $41 \%$ das crianças relataram não deglutir o creme dental e $36 \%$ responderam que deglutiram poucas vezes, enquanto $23 \%$ afirmaram ter ingerido diversas vezes.

Ademais, aliado ao alto nível de ingestão de creme dental, outro fator de risco para o desenvolvimento da fluorose dentária é a quantidade de dentifrício colocado na escova. Quanto maior for a quantidade de dentifrício utilizada pela criança, maior será a chance de deglutição da mesma e uma posterior ingestão acima do necessário (CALDARELLI PG, et al., 2016; LIMA-ARSATI YBO, et al., 2018). 
Neste contexto, Lima-Arsati YBO, et al. (2018) relataram em seu estudo que todos os voluntários colocaram na escova uma quantidade de creme dental superior ao recomendado para a faixa etária $(0,1 \mathrm{~g})$, sendo obtida uma média de 0,47 g, uma quantidade, aproximadamente, cinco vezes maior do que o valor sugerido. Refutando esses resultados, Miranda FP e Cericato GO (2016) constataram que $59 \%$ das crianças aplicava uma quantidade moderada de creme dental, enquanto $36 \%$ aplicava o dentifrício em toda a escova, o que não é recomendado.

A supervisão das crianças durante a escovação é associada ao fato de que crianças com idades menores não sabem realizar a eliminação completa do dentifrício, diante disso, elas podem apresentar algumas dificuldades motoras no controle da deglutição. Dessa forma, para obter um maior controle da ingestão do creme dental pelas crianças, é recomendada a presença de um responsável para dispensar o dentifrício na escova, sendo considerada como uma medida preventiva que visa evitar o risco de fluorose dentária (JAGHER AC, et al., 2016).

A falta de conhecimento sobre os cuidados necessários com a higiene bucal é um dos fatores a ser considerado, uma vez que, a informação, embora esteja disponível nas mídias, não chega a todas as camadas da população de maneira uniforme, e dificilmente é aprendida de modo a produzir conhecimento e autonomia em relação aos cuidados com a saúde bucal. É comprovado que existe a possibilidade de prevenção e controle de doenças bucais que ocorrem devido ao biofilme através da modificação de seus fatores etiológicos (ANTONIO LP, et al., 2015).

Segundo a pesquisa realizada por Damião LS, et al., (2010), a maioria dos pais e responsáveis relataram utilizar uma pequena quantidade de dentifrício fluoretado durante a escovação das crianças. No entanto, o estudo de Jagher AC, et al. (2016) observou que que a quantidade de creme dental colocada na escova era superior a recomendada para a faixa etária, onde a dose mencionada pelo responsável ou pela criança foi menor do que a colocada na escova.

É importante pontuar que as mães exercem uma influência especial nas questões relacionadas à saúde da criança, uma vez que, elas são as que mais acompanham o tratamento dos filhos. Por isso, percebe-se a necessidade de sugerir trabalhos de prevenção em saúde bucal voltados principalmente para as mães das crianças (MARTINS CLC e JETELINA JC, 2016).

A educação e, principalmente, a motivação das crianças são estratégias essenciais no processo de promoção de saúde bucal, devendo serem realizadas constantemente e de forma dinâmica. Com a realização de ações educativas em saúde, nota-se um aumento no nível de conhecimento das crianças sobre o assunto e gera melhorias nos hábitos e cuidados com a saúde bucal (CRUZ MCC, et al., 2015; GARCIA PPNS, et al., 2013; RIBEIRO DG, et al., 2009). Esse desenvolvimento de atividades educativas em saúde buccal, ainda no período da infância, são de extrema importância para a formação de hábitos saúdaveis pelo indivíduo, já que as crianças apresentam uma capacidade maior de assimilar novos conhecimentos e aplicá-los durante toda a vida, favorecendo o desenvolvimento de futuros adultos com qualidade na saúde oral e geral (CRUZ MCC, et al., 2015; SANTOS NML, et al., 2010)

O processo educativo em saúde na infância proporciona trocas de vivências e experiências entre os indivíduos, utilizando-se de diversas métodos e recursos que estimulem o raciocínio da criança ao assunto apresentado. Assim, métodos audiovisuais dinâmicos como o teatro, os vídeos, os panfletos e as gincanas devem ser trabalhados com esse público, podendo ser utilizado em associação com os métodos educativos tradicionais, como a palestra (ANTONIO LP, et al., 2015). Em seu estudo, Garcia PPNS et al. (2013) buscou avaliar o efeito de um instrumento educativo em saúde bucal aplicado através de diferentes métodos. Através de seus resultados foi possível concluir que o método de autoinstrução associado à discussão e debate do conteúdo proporcionou um maior entendimento do assunto.

A partir desse teste, observou-se, estatisticamente, diferenças entre os grupos, onde o método associado se mostrou mais eficaz. Dessa forma, acredita-se que há uma dificuldade na assimilação das orientações, realizadas pelos profissionais, quando elas são apenas verbais, por parte dos pais ou responsáveis e pelas crianças, sendo importante que os mesmos busquem métodos alternativos como, por exemplo, as demonstrações visuais e as ilustrações para elucidar o conhecimento para os seus pacientes e familiares acerca das quantidades adequadas de creme dental para cada faixa etária (JAGHER AC, et al., 2016). 
Muitas vezes, os métodos mais sofisticados podem ser menos eficientes do que os mais simples, se não forem utilizados de maneira correta. Desse modo, é preferível utilizar o método com o qual se esteja mais familiarizado e capaz de extrair toda sua eficiência (ANTONIO LP, et al., 2015).

\section{CONSIDERAÇÕES FINAIS}

De acordo com os resultados encontrados nesta pesquisa observou-se que não há diferença estatística em relação ao método educativo utilizado entre os grupos de crianças, onde, se qualquer uma dessas intervenções forem utilizadas, terão um resultado positivo sobre a quantidade adequada de creme dental a ser utilizada pelas crianças. Entretanto, quando avaliados por outro teste, o método educativo associado apresentou melhores resultados para a amostra selecionada.

\section{REFERÊNCIAS}

1. ANTONIO LP, et al. Avaliação de diferentes métodos educativos em saúde bucal em crianças na faixa etária de 7 a 10 anos de idade. Revista da Faculdade de Odontologia-UPF, 2015; 20(1): 52-58.

2. BALLESTRERI R, et al. Hábitos de saúde bucal em crianças internadas no Hospital da Crianca no município de Chapeco, Santa Catarina, Brasil. Revista Da Faculdade De Odontologia - UPF, 2016; 21(3): 300-305.

3. CASTILHO LS, et al. O papel do dentifrício sem flúor na ocorrência da cárie dentária entre pacientes com deficiências de desenvolvimento até os seis anos de idade. Revista Brasileira de Extensão Universitária, Chapeco, 2016; $7(2): 121$ - 127.

4. CURY JA, et al. Effect of dentifrice containing fluoride and/or baking soda on enamel demineralization/ remineralization: na in situ study. Caries Res 2001; 35(2):106-10.

5. CRUZ MCC, et al. Método de educação em saúde bucal para estudantes. Archives of Health Investigation, 2015; 4(5):4654.

6. CALDARELLI PG, et al. Contribuição da água e dentifrício fluoretado na prevalência de cárie e fluorose dentária: uma abordagem baseada em evidências. Journal of the Health Sciences Institute, 2016;34(2):117-122.

7. CAMATTA IB, OLIVEIRA CS. Avaliação do conhecimento das famílias sobre a higienização bucal e o uso domiciliar de fluoretos em crianças de uma faculdade particular do Espírito Santo. Journal of Health \& Biological Sciences,2019; 7(2):166171.

8. DUQUE AD, et al. Strategies for the prevention of periodontal disease and its impact on general health in Latin America. Section III: Prevention. Brazilian Oral Research, 2020; 34(Supl. 1): e025.

9. DAMIÃO LS, et al. Uso de dentifrício fluoretado e hábitos de escovação em crianças de 6 a 36 meses de idade. Revista Odontológica do Brasil Central, 2010; 19(51): 295-300.

10. FILHO PA, et al. Fatores associados a cárie dental e doença periodontal em indígenas na América Latina: revisão sistemática. Revista Panamericana de Salud Pública, 2014; 35(1): 67-77.

11. GARCIA PPNS, et al. Educação em Saúde: Efeito de um Método de Auto-Instrução Sobre os Níveis de Higiene Oral em Escolares. Pesquisa Brasileira em Odontopediatria e Clínica Integrada, 2009; 9(3):333-337.

12. JAGHER AC, et al. AvaliDos ação da utilização de dentifrício fluoretado em crianças. Revista da Faculdade de OdontologiaUPF, 2016; 21(1): 37-42.

13. LAZZARIN HC, et al. Avaliação do perfil de prescrição de dentifrícios fluoretados por cirurgiões dentistas em crianças menores de 06 anos. Conscientiae saúde, 2018; 17(1): 32-40.

14. LIMA MNT, et al. Proposta de patente de Dosador de dentifrício fluoretado para uso infantil. Revista Pró-UniverSUS, 2018; 9(2): 95-102.

15. LOPES LM, et al. Indicadores e fatores de risco da cárie dentária em crianças no Brasil - uma revisão de literatura. RFO, 2014; 19(2): 245-251.

16. LIMA-ARSATI YBO, et al. Exposição a fluoreto por crianças na faixa etária crítica para fluorose dentária, residentes no semiárido brasileiro. Ciência \& Saúde Coletiva, 2018; 23(4): 1045-1054.

17. MAGALHÃES AC, et al. Uso racional de dentifrícios. Revista Gaúcha de Odontologia, 2011; 59(4): 615-625.

18. MARTINS CLC, JETELINA JC. Conhecimento dos pais sobre saúde bucal na infância e a relação com o motivo da consulta odontológica. Journal of Oral Investigations, 2016; 30(1): 27-33.

19. MIRANDA FP, CERICATO GO. Fluorose dentária em escolares de 12 anos do município de lbirapuitã (RS). Journal of Oral Investigations, 2016; 5(2):16-22.

20. OLIVEIRA CC, et al. Conhecimento dos cirurgiões-dentistas da estratégia saúde família quanto à utilização do dentifrício fluoretado em crianças. Revista Intercâmbio, 2018; 11: 174-186.

21. RIBEIRO DG, et al. Avaliação de um método educativo em saúde buccal aplicado em escolares de ensino público, 2009; 45(3): 154-159.

22. SILVA MF, et al. O que os estudantes de medicina aprendem sobre creme dental infantil?. Saber Digital, 2019; 12(1): 102122.

23. SILVA-JUNIOR MF, et al. Condição de saúde bucal e motivos para extração dentária entre uma população de adultos (2064 anos). Ciência \& Saúde Coletiva, 2017; 22(8): 2693-2702.

24. SOUSA JB, et al. Saúde bucal na escola: Um estudo sobre atividades de educação em saúde para estudantes. Jornada Odontológica dos Acadêmicos da Católica, 2017; 3(1): 2693-2702.

25. SANTOS NML, et al. O Impacto do Programa Preventivo-Educativo da Unidade de Saúde da Família Viçosa na Saúde Bucal de Escolares. Revista da Faculdade de Odontologia de Porto Alegre, 2010; 50(2):15-19.

26. SILVA DAS, et al. Risco de intoxicação em ambiente doméstico por creme dental fluoretado. Revista Pró-UniverSUS. 2017; $8(1): 62-66$. 